\title{
Importance of Practical Relevance and Design Modules in Electrical Circuits Education
}

\author{
doi:10.3991/ijoe.v7i2.1646 \\ R. A. Amarin ${ }^{1}$, I. Batarseh ${ }^{2}$, K. B. Sundaram ${ }^{1}$ and A. Weeks ${ }^{1}$ \\ ${ }^{1}$ University of Central Florida / Department of Electrical Engineering and Computer Science, Orlando, USA \\ 2 on Professional Development Leave at Princess Sumaya University for Technology, Amman, Jordan
}

\begin{abstract}
The interactive technical electronic book, TechEBook, currently under development at the University of Central Florida (UCF), provides a useful tool for engineers and scientists through unique features compared to the most used traditional electrical circuit textbooks available in the market. TechEBook has comprised the two worlds of classical circuit books and an interactive operating platform such as iPads, laptops and desktops utilizing Java Virtual Machine operator. The TechEBook provides an interactive applets screen that holds many modules, in which each had a specific application in the self learning process.
\end{abstract}

This paper describes two of the interactive techniques in the TechEBook known as, Practical Relevance Modules (PRM) and Design Modules (DM). The Practical Relevance Module will assist the readers to learn electrical circuit analysis and to understand the practical application of the electrical network theory through solving real world examples and problems. The Design Module will help students design reallife problems. These modules will be displayed after each section in the TechEBook for the user to relate his/her understanding with the outside world, which introduces the term me-applying and me-designing, as a comprehensive full experience for self or individualized education. The main emphasis of this paper is the PRM while the DM will be discussed in brief. A practical example of applying the PRM and DM features is discussed as part of a basic electrical engineering course currently given at UCF and results show improved student performances in learning materials in Electrical Circuits. In the future, such modules can be redesigned to become highly interactive with illustrated animations.

Index Terms-Practical Relevance Module, Design Module, Tool, Electrical Circuits, Interactive book.

\section{INTRODUCTION}

The interactive TechEBook consists of 16 chapters, a total of 75 sections representing typical content for the introductory circuit course at most universities in the world. Each section discusses a new theory and concept that are supported with examples and problems [1]. Different topics are presented in discussion text material that provides full understanding of the concept. At the end of each section, QuizMe Modules are provided to quiz the students' understanding of the section [2]. Also, TutorMe Modules are intended to help understanding basic concepts in a step-by-step manner [3]. Design Modules (DM) are intended to help students develop their ability to design real life problems, and to link the theories they study in books with real design challenges, while the Practical
Relevance Modules (PRM) are set to enhance the student thinking about real life problems and also teach students how to relate the theories they have learned with practical applications.

\section{MOTIVATION}

At the University of Central Florida, efforts are underway to develop the full content of the TechEBook using the above attributes of each section. Practical Relevance and Design Modules are two major components of the TechEBook currently being developed through partial funding from NSF. The main objective of including these modules is to provide engineering students in the electrical circuit classes with a better understanding of the practical application for the electrical network theory through solving and designing real time examples.

The DM includes solving for different circuit parameters to satisfy a certain condition. This will improve the students' ability to design the circuit in many different ways to enhance their engineering sense. The Practical Relevance exercises will be dynamic with animation features and the ability for students to enter various values for formulas, which in turn will be dynamically used to convey a variety of results for the specific topics.

Currently, the major circuit analysis books have very limited real world examples and problems. In fact, almost none of the existing books include interactive and animated real world problems. In order to help students understand the application of the circuit theory presented in the classroom, it will be advantageous if the presented problems and examples are based on existing product in the market. Therefore, the PRM will:

1. Present real world applications.

2. Relate the application to the circuit theory being studied.

3. Relate the application to real products in the market.

4. Provide interactive applet to show how the application works under different parameter variations.

Almost all of today's consumer electronics products in the market are designed based on a technical area in the field of electrical engineering. These areas include electrical circuits, electromagnetics, analog electronics, controls, digital electronics, digital signal processing, digital and analog filters, digital and analog communications, computer architecture, sensors, networking, power electronics, power systems, optics and photonics, lasers, antennas and satellites, machines, and drives. Ultimately, the electrical engineering concepts that use the technical areas listed above are used to develop new technology 
and products: cell phones, MP3 players, CD players, DVD players, TVs, radios, computers, calculators, displays, cars, planes, trains, motorcycles, ovens, refrigerators, dishwashers, microwaves, power outlets, air conditioners, power supplies, lamps, and lawnmowers. Each of the above products can be used to generate many realworld examples that use the technical areas mentioned above.

\section{PRM IMPLEMENTATION PLAN}

The PRM is still under development at UCF. To accomplish the PRM objectives, this project is implemented over two phases as follows:

Phase I: Develop the PRMs for all sections.

Phase II: Develop the Interactive Applets for the PEMs developed in Phase I. Each is discussed next.

\section{A. Phase I: Implementation Plan}

During this phase, about 75 real world examples were developed, where each PRM is a complete stand alone module. Each PRM presents an application for the electrical circuit concepts in each section. Hence, each section is based on technical areas in Electrical Engineering. Ultimately, the electrical engineering concepts that use the technical areas listed in Section II are used to develop new technology and products.

\section{B. Phase II: Interactive and Animation Applets}

The second phase of the project will be to create interactive applets that will help students:

1. Vary certain circuit parameter and observe the desired outcome.

2. Have better insight on the application and how the system functions.

3. How the design process affects the way the application/system functions.

4. Use the applet for design purposes.

5. Confirm students understanding of the application.

The second phase will include the following items:

1. Review the up-to-date open market published material whether printed or web based.

2. Identify what applications in the TechEBook can be developed as interactive applets.

3. Develop learning objectives for each applet.

4. Identify what parameters will vary and how.

5. Provide detailed description on how the application works.

6. Provide detailed help menu explaining how each applet works. Give step-by-step instructions.

7. Final written report and software tool of the developed applet modules.

\section{PRM ARCHITECTURE}

To generate a real world Practical Relevance problem that can be tied to an existing product and to show the technical theory being studied, the block diagram in Fig. 1 provides a high level example of the major PRM components. After each section of the TechEBook, the Topic of the PRM will be presented, followed by the Example description, then the real world Application along with the Circuit Model and finally the Product that uses the specified circuit.

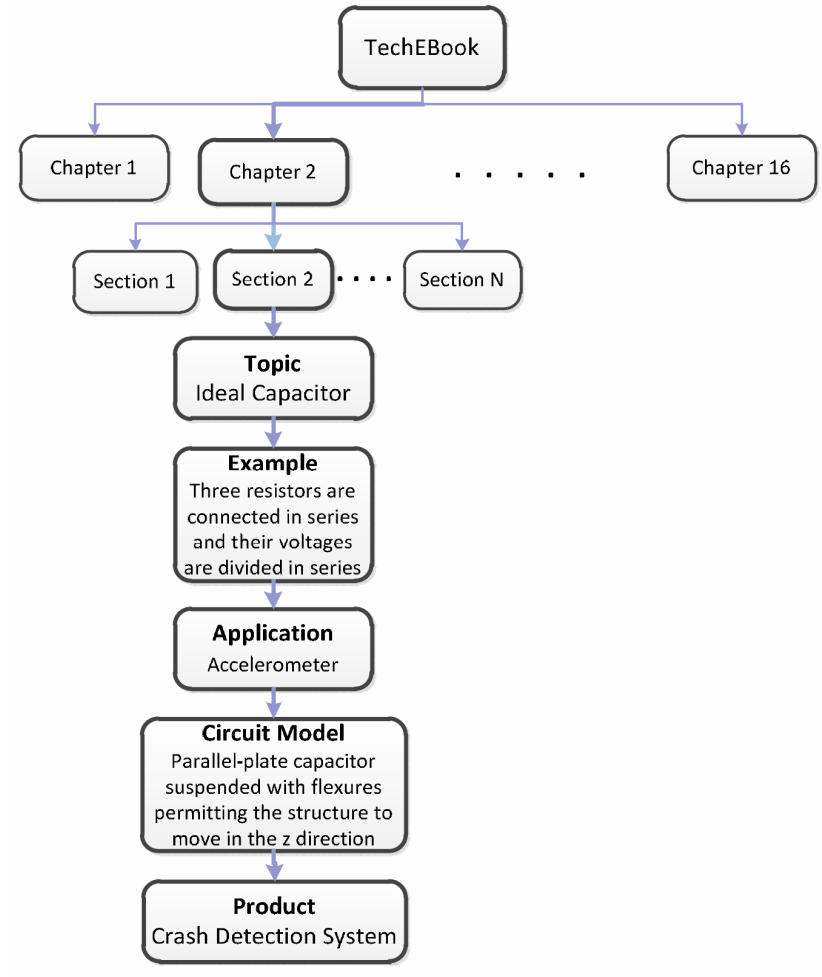

Figure 1. PRM architecture

The major learning aspects include:

1. The theory of the circuit concept provided by the section.

2. Working out a real world example.

3. The knowledge about where this new concept is used in real systems.

4. How to obtain from the real world system an equivalent circuit model.

5. Where and how the circuit concept (theory) is being used in real world systems or products.

6 . In the process, students will learn about practical devices and components used in real products: Resistors, capacitors, inductors, voltage sources, fuses, diodes, transformers, LED, Transistors (all kinds), switches, Op Amps, batteries, instrumentation, light bulb, digital counters, seven segment display, zener diode, motors, three phase transformer, audio amplifier, switching regulator (power supplies), crystal oscillators ... etc. This includes model name, type, shape, manufactures ... etc.

7. The ability to locate and read specification data sheet related to these devices and components.

\section{PRM EXAMPLE}

For a 'Section' in the TechEBook that describes the Ideal Capacitor concept, a crash-detection system was selected as the commercial 'Product' to describe the functionality and theory behind the ideal capacitor.

The PRM starts with a brief discussion about the topic of passengers' safety on roads and the safety systems that are developed to protect the occupants. Safety systems can be either active by preventing accidents or passive by protecting the occupants against serious or even fatal injuries. An example of a passive safety system is the car 
airbag, which is the PRM example covered in this paper. The airbag system is activated upon crash detection. Crashes can be detected using a special sensor called 'accelerometer', which is the 'Application' of this PRM.

Accelerometers measure acceleration, which is the rate of change of velocity. In case of crash, sudden change in velocity takes place, which results in an increased acceleration that will exceed a certain threshold and then the crash is detected by the system. For the crash detection systems, the precision of the sensor is crucial in saving lives.

The 'Circuit Model' is explained next. The capacitor between conductors is a function of conductor size $A$, spacing $z$, and the permeability $\varepsilon$ of the material between the conductors. Fig. 2 shows a simple parallel-plate capacitor suspended with flexures permitting the structure to move in the $z$ direction.

A more depth explanation with images and equations to better understand the concept is provided in the real example. Fig. 3 shows a simple electrical model of the interface circuit for the crash detection sensor. The sense capacitor is placed in series with a reference capacitor $C_{r e f}$. Several parasitic capacitors $C_{P i}$ are also used. In the real example, the output voltage of the circuit $V_{o}$ is calculated, which represents the sensed acceleration.

A 'Numerical Example' follows the circuit model to illustrate the understanding of the crash detection systems and a complete analysis of the problem is performed. Finally, a set of references are provided for convenience.

This example is accompanied with dynamical animation features for a better insight on the application and how the system functions. Two examples are given in Fig. 4 to show the difference between a low speed collision (10 MPH - 16 KMH) and a high speed collision (50 MPH $-80.5 \mathrm{KMH})$. When playing the applet, the car starts moving towards the wall with a $50 \mathrm{MPH}(80.5$ $\mathrm{KMH}$ ) speed and the moment it crashes, the accelerometer will sense the increased acceleration above the threshold, and therefore the airbag will inflate to protect the occupants. For a car moving at $10 \mathrm{MPH}(16 \mathrm{KMH})$, the threshold is not exceeded, and hence the airbag will not inflate.

\section{EXPERIMENTAL RESULTS}

As part of developing the TechEBook, a survey of 58 students of different majors from the Electrical Networks (EEL 3004) class at the University of Central Florida was conducted and results were analyzed. A set of problems were developed with design and practical relevance emphasis. The focus of this survey was to study the influence of practicing these modules and how it can assist the students in learning electrical circuit analysis. The class was divided into two groups (A and B) consisting of 29 students each. Group A was provided with ten sample problems from the developed Design Module and Group B was provided with ten sample problems from the Practical Relevance Module. Each of the groups was requested to solve the problems and to submit as an assignment for the course. In the final examination, one question from Design Module and one question from Practical Relevance Module were included to test whether the prior exposure of the developed module really helped the students' learning capability. Group A had an average score of 8.69/10 in the Design Problem category and an average score of $3.62 / 10$ in the Practical Relevance category. On the other hand, Group B showed an average of $6.69 / 10$ in the Design Problem category and an average of 5/10 in the Practical Relevance category as shown in Fig. 5. Preliminary analysis indicated that prior opportunity to practice the relevant modules improved their performances in learning materials in Electrical Circuits.

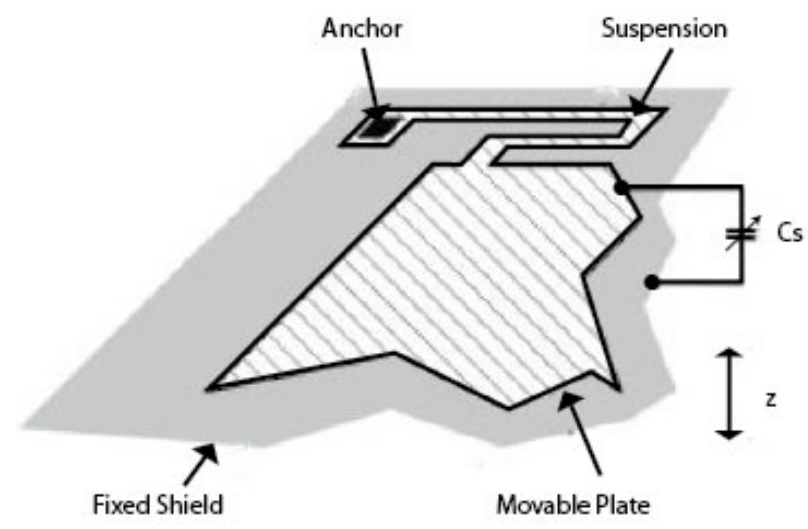

Figure 2. Parallel-plate capacitor with suspension

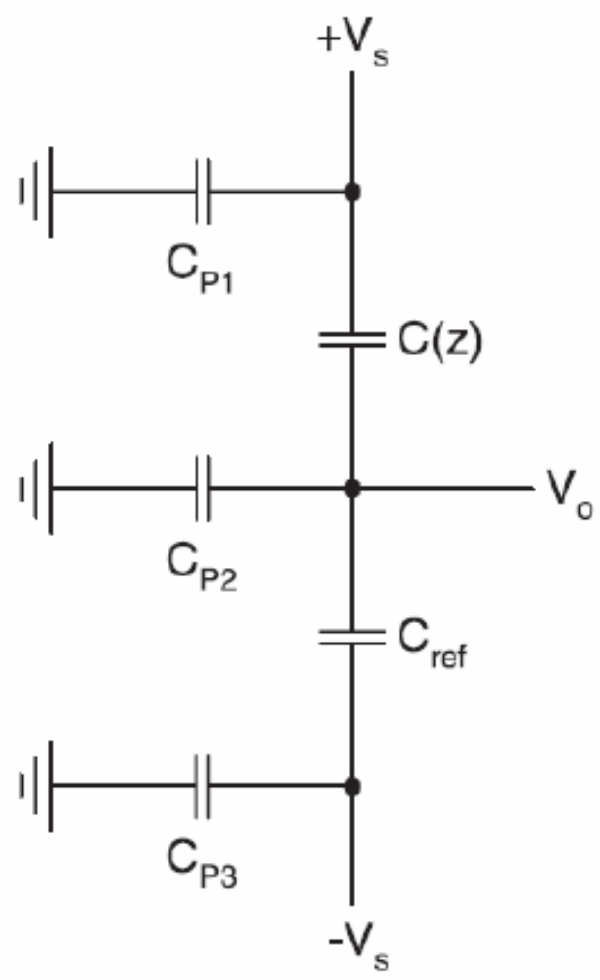

Figure 3. Electrical equivalent circuit for a single-ended parallel plate capacitive transducer interface 

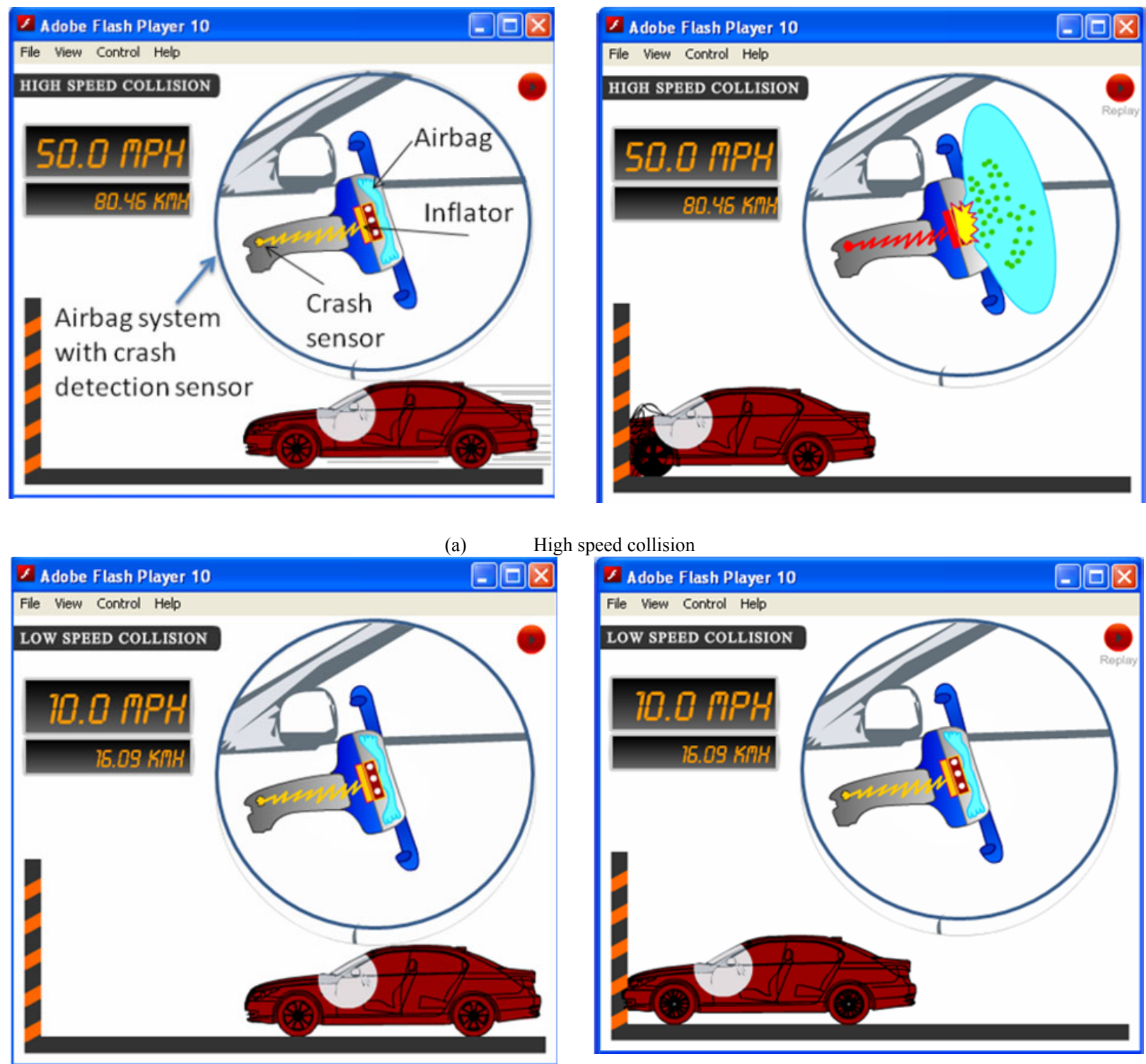

(b) Low speed collision

Figure 4. PRM interactive applet example

VII. SUMMARY

There is no doubt that learning is the world's new language of communication and knowledge delivery, and TechEBook has been used to translate streaked old circuit basic books to a new well developed and easy to use tool. This paper, addresses the TechEBook Practical Relevance and Design Modules in particular. It presents a new electronic, interactive and adaptive method to maximize the students learning experience, in their self learning process (me-applying, me-designing) and increase their understanding in any given topic.

The main purpose of the PRM and DM techniques is to give a well understanding and a fair knowledge about relating theories to real world applications, and to enhance the engineering sense within students. The overall assessment of 58 students who took the PRM and DM resulted in an average score of $43 \%$ and $77 \%$, respectively. Most students welcomed the new tools, which reflected the importance of these on-line modules.

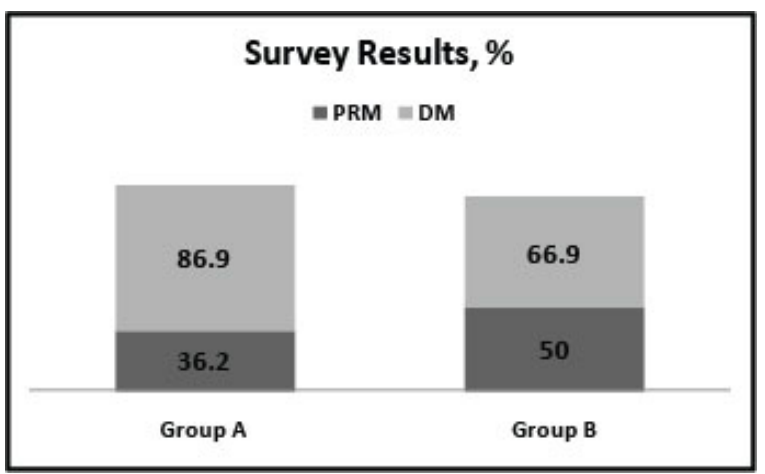

Figure 5. Survey results

\section{REFERENCES}

[1] Batarseh, Ghaith Haddad, Rawad Haddad and Rashad Oreifij, 'Interactive Electronic Book Operating Systems And Methods,' United States Patent 20080222552. 
[2] Ruba A. Amarin, Feras Batarseh and Issa Batarseh, 'Adaptive Electronic Quizzing Method for Introductory Electrical Circuit Course', International Journal of Online Engineering, 2009.

[3] Ghaith Haddad, Gustavo Gamboa and Issa Batarseh, 'Interactive Electrical Circuit Tutoring Tool. eTutor,' 2008 ASEE Southeast Section Conference, Memphis, TN, April 2008.

[4] Feras Batarseh, Moataz Abdelwahab and Issa Batarseh, 'Quiz MeAn Interactive Learning Tool with Application to Electrical Circuits,' ASEE SE Section Annual Conference - Marietta, GA, April 2009.

\section{AUTHORS}

Ruba A. Amarin is with the University of Central Florida, Orlando, FL 32816 USA (e-mail: ramarin@mail.ucf.edu). Ruba received the B.S. degree in Electronic Engineering from the Princess Sumaya University for Technology, Amman-Jordan in 2004; she afterwards received her M.Sc. degree in Electrical Engineering, Tele-Communication track from the University of Central Florida, Orlando in 2006. In 2010, Ruba received her $\mathrm{PhD}$ in Electrical Engineering, conducting research in Satellite and Remote Sensing under the supervision of Dr. Linwood Jones.

Issa Batarseh is currently on professional development leave at the Princess Sumaya University for Tech- nology (PSUT) (e-mail: batarseh@mail.ucf.edu). He received the B.S. degree in Computer Engineering and the M.S. and Ph.D. degrees in Electrical Engineering from University of Illinois, Chicago, in 1983, 1985, and 1990, respectively. He was a Visiting Assistant Professor of Electrical Engineering at Purdue University, Calumet City, IN, from 1989 to 1990 before joining the Department of Electrical and Computer Engineering at the University of Central Florida, in 1991. He has more than 14 U.S. patents, and more than 50 refereed journal and 200 conference publications.

K. B. Sundaram is with the University of Central Florida, Orlando, FL 32816 USA (e-mail: sundaram@mail.ucf.edu).

A. Weeks is with the University of Central Florida, Orlando, FL 32816 USA (e-mail: weeks@mail.ucf.edu).

This work is partially funded by NSF - CCLI Award Number: 0837364 No.

This article is an extended version of a paper presented at the IEEE EDUCON20211 Conference, held from April 4th-6th, 2011, in Amman, Jordan. Received April 25 ${ }^{\text {th }}$, 2011. Published as resubmitted by the authors April $30^{\text {th }}, 2011$. 